\title{
L'INDUSTRIE LAITIERE DANS LE MONDE
}

\author{
par \\ G. GÉNIN
}

Ingénieur E. P. C.

\section{La fabrication mondiale de la poudre de lait}

L'importance considérable qu'a prise la fabrication de la poudre de lait apparaît dans les chiffres qui montrent le développement de cette fabrication depuis la fin de la guerre. En 1956, la production totale mondiale de poudre de lait entier et de poudre de lait écrémé, a été supérieure à 1,1 million de tonnes, soit 5 fois plus élevée que celle d'avant guerre et supérieure de $60 \%$ à celle de la période 1948-1952. Sept pays ont produit $90 \%$ de cette fabrication totale en 1956, les Etats-Unis arrivant largement en première place avec un pourcentage de $64 \%$. Le Royaume-Uni a remplacé les Pays-Bas à la seconde place et on a enregistré une légère réduction de la production canadienne et australienne et une augmentation de celle de la Nouvelle-Zélande. Le Japon a créé cette industrie depuis la guerre et sa production atteint celle du Danemark et de la France.

L'augmentation de la production a surtout été sensible pour la poudre de lait écrémé, par suite du prix plus bas du produit et d'un développement de ses applications. L'augmentation de cette production a surtout été sensible en Angleterre, au Canada, en NouvelleZélande, en Australie et en Belgique.

Les exportations qui, avant la guerre, représentaient $15 \%$ de la production totale mondiale, ont atteint $30 \%$ en 1956 , ce qui a stimulé la production. Les Etats-Unis, les Pays-Bas, la NouvelleZélande et l'Australie sont les principaux pays exportateurs, alors que l'Angleterre est le premier pays importateur.

Dans la majorité des pays producteurs, la consommation de lait en poudre a augmenté d'une façon sensible. En NouvelleZélande et en Allemagne, elle est 9 fois celle d'avant-guerre; en Suède, 6 fois; aux Etats-Unis et au Canada, 3 fois; en Belgique et en Angleterre, 2,5 fois. On note simplement une diminution de cette consommation en Suisse et au Danemark. Ce sont les EtatsUnis et la Nouvelle-Zélande qui enregistrent la plus forte consommation avec $2 \mathrm{~kg} .8$ par habitant et par an.

\section{Industrie mondiale de la caséine}

Le développement de la production laitière a conduit les producteurs à réaliser un effort important pour l'utilisation des sous- 
produits de cette industrie et la fabrication de la easéine, sousproduit du lait écrémé a pris depuis une dizaine d'années un important développement, ce produit trouvant d'intéressantes applications dans les industries suivantes : fabrication du papier couché, préparation de peintures à l'eau, fabrication de colles, fabrication de plastiques ininflammables, production de fibres textiles, fabrication de produits pharmaceutiques, fermentation du vin, transfusion du sang, etc. Cependant, la caséine s'est trouvée placée en concurrence dans ces applications avec de nombreux produits synthétiques, ce qui a conduit à une stabilisation des prix et en même temps a freiné un peu le développement de cette industrie.

Néanmoins, alors qu'avant la guerre, la production mondiale était de 77.000 tonnes, elle a atteint en 1955 , un total de 93.000 tonnes, soit une augmentation de l'ordre de $20 \%$. On a enregistré également de profonds bouleversements dans l'ordre de classement des pays producteurs. En 1938, les Etats-Unis étaient le principal pays producteur avec une production de 22.000 tonnes, or en 1955 , la production n'a pas dépassé 1.400 tonnes, le lait écrémé, matière première de la fabrication de la caséine étant de préférence employé pour la fabrication de la poudre de lait écrémé. En 1938, la République argentine occupait la seconde place. Pendant et après la guerre, la production de caséine dans ce pays s'est régulièrement développée, et elle dépasse aujourd'hui 40.000 tonnes. Vient ensuite la France qui en 1938, avait une production de 16.500 tonnes, pendant la guerre, et dans les années qui ont suivi immédiatement les hostilités, cette production s'est abaissée, mais elle s'est à nouveau élevée à 17.800 tonnes en 1955 .

La Nouvelle-Zélande et l'Australie sont devenues aujourd'hui d'importants producteurs de caséine. En Nouvelle-Zélande, la production est passée de 3.500 tonnes en 1938 à 14.100 tonnes en 1956 et en Australie elle est passée de 2.800 tonnes en 1938 à 9.500 tonnes en 1956, la caséine constituant dans ces deux pays un sous-produit de la fabrication du beurre.

En Italie, on enregistre un développement de la production et des importations de caséine, la production passant de 2.000 tonnes en 1938 à 6.400 en 1956 , la caséine étant utilisée surtout pour la fabrication de la fibre synthétique Lanital, fibre également fabriquée aux Pays-Bas et en Angleterre, surtout à une époque où la laine avait atteint des cours très élevés.

$85 \%$ des exportations mondiales de caséine sont alimentées par la production de la République argentine, de France, d'Australie et de Nouvelle-Zélande, ces exportations ont passé de 
30.000 tonnes en 1938 à 77.800 tonnes en 1956 , l'Argentine occupant la première place avec un pourcentage de $45 \%$.

\section{ANGLETERRE}

\section{Eveilleur d'acide résistant aux bactériophages}

L'une des principales difficultés que l'on rencontre en Angleterre dans la fabrication du Cheddar et du Cheshire, résulte de ce que certains lots de lait subissent une fermentation ralentie, la transformation du lactose en acide lactique étant très lente. On admet que ce phénomène est dû à la présence dans les éveilleurs d'acide de certains bactériophages qui réduisent l'activité de ces éveilleurs.

La Fisons Milk Products Ltd fabrique aujourd'hui sur une base commerciale un nouveau milieu de culture destiné à la préparation des éveilleurs d'acide, présentant l'avantage d'être parfaitement résistant à l'action des bactériophages. Ce produit, dénommé Cockade P. R. M. est dû aux travaux d'un savant autrichien, le Dr Bruno ReITER, et ce produit a été présenté pour la première fois au Congrès international de la laiterie de Rome, en 1956. Il a été utilisé expérimentalement en Angleterre, en Nouvelle-Zélande, en Australie et aux Etats-Unis et on estime que son emploi pourrait réduire totalement les difficultés rencontrées dans la fabrication du fromage et qui provoquent des pertes s'élevant à $5 \%$ de la production mondiale.

Le produit est préparé en partant de lait privé d'ions calcium, il est vendu sous la forme d'un produit déshydraté, auquel on ajoute de l'eau exempte de sels de calcium au moment de son utilisation.

\section{Nouvel appareil pour la fabrication de parts de crème glacée}

L'A. P. V. Co vient d'ajouter à la liste du matériel construit pour l'industrie laitière une nouvelle machine destinée à la confection des parts individuelles de erème glacée. Cet appareil, vendu sous le nom de Rollo Ice Cream Bar Freezer est dû aux travaux de la firme danoise Hoyer Manufacturing Co, et suivant les modèles, il permet de confectionner 6.000 à 12.000 parts de crème glacée montées sur support en bois et recouvertes d'un enduit de chocolat.

L'appareil comporte un dispositif de refroidissement et de moulage des parts de crème, ainsi qu'une installation de stérilisation. Il peut également servir à conserver les parts déjà fabriquées, lorsque la machine n'est pas en fonctionnement.

\section{Nouveau type de fromage}

Le Scottish Milk Marketing Board vient de mettre au point la 
fabrication d'un nouveau fromage du type Dunlop, dénommé "Torrylinn". Il s'agit d'un fromage sans croûte qui est livré en pains d'environ 18 kilogrammes et qui renferme dans toute sa masse une teneur en humidité parfaitement répartie. Ce fromage est enveloppé dans une pellicule d'emballage dénommée Pukkafilm et porte le nom du produit imprimé sur cet emballage. En Ecosse, la vente de ce produit est assurée par la Compagnie des Fabricants de fromages écossais, et en Angleterre la fabrication est assurée par la Mauchline Creamery, sous le nom de Mauchline.

A l'exposition laitière de Londres, qui s'est tenue récemment à Olympia, les fabricants de fromage avaient exposé un fromge géant de ce type, pesant près de 500 kilogrammes et pour la fabrication duquel il avait fallu réaliser une presse spéciale.

\section{Procédé de traitement des bouteilles en verre en vue de réduire leur casse et leur détérioration}

La Clarke Built Ltd est devenue le seul concessionnaire pour la Grande-Bretagne et l'Europe continentale, du procédé de traitement des bouteilles en verre Thatcher NU-Glass qui permet de réduire la casse et la détérioration des bouteilles en verre utilisées pour la livraison du lait, de la bière et des boissons gazeuses.

On a constaté en effet que si la casse des bouteilles par chocs est relativement réduite, par contre, les rayures qui se produisent lorsque les bouteilles sont manipulées au cours de leur remplissage, de leur livraison et de leur nettoyage, provoquent un affaiblissement des bouteilles et conduisent à une rupture sous l'influence d'une simple manutention ou de changements de température.

Cet inconvénient peut être considérablement réduit et la casse réduite d'environ $50 \%$ en projetant au moyen d'un pistolet, à la surface extérieure des bouteilles, une pellicule d'huile contenant en suspension certains dérivés hydrocarbonés et de la silice. Le verre se trouve ainsi protégé par une sorte de pellicule lubrifiante qui réduit les rayures et la casse des bouteilles. Dans le mélange projeté, c'est la silice qui assure l'adhérence du revêtement lubrifiant sur le verre, mais il importe cependant que ce traitement de protection soit fréquemment répété et pratiquement après chaque opération de nettoyage et de remplissage.

\section{Nouveau papier d'emballage résistant aux moisissures}

Sous le nom de Nevermold 101, la William Palfrey Ltd présente à sa clientèle un nouveau papier d'emballage résistant aux moisissures qui est le fruit de plusieurs années de recherches en laboratoire. Des essais ont montré que ce papier ne subit aucune altération lorsqu'il a été recouvert pendant quatorze jours d'un milieu 
de culture contenant ou ne contenant pas de dextrose et ensemencé en moisissures, telles que Chaeromium globosum, Aspergillus terreus et Aspergillus niger. Ce papier peut également être enterré pendant plus de 30 jours sans diminution de ses qualités mécaniques, ses qualités fongicides ne sont pas altérées après un lavage à l'eau courante d'une durée de 24 heures ou après un chauffage en étuve à $80^{\circ}$ pendant 48 heures. On a enfin pu établir, qu'il n'est pas détruit par l'action des rayons ultra-violets, sa toxicité est extrêmement faible et il ne présente aucune odeur. L'emploi de ce papier présente donc un grand intérêt pour l'emballage de produits alimentaires nombreux.

\section{Nouveau procédé d'emballage du Cheddar}

La Kraft Foods Ltd présente un nouveau mode d'emballage du Cheddar sous le nom de Red Barrel. La pellicule d'emballage utilisée dans ce cas est constituée par une feuille de cellophane protégée par une pellicule de cire. Cet emballage, dénommé Pukkafilm, a fait l'objet de recherches entreprises par le National Institute for Research in Dairying qui ont montré qu'un fromage emballé dans cette pellicule peut se conserver pendant une longue durée lorsqu'il est stocké à une température suffisamment basse.

Le fromage ainsi emballé est livré sans croûte. L'emballage empêche pratiquement toute perte de poids du produit et assure sa protection efficace contre la poussière et les bactéries. Si le fromage est entamé, il peut être à nouveau réemballé dans le même emballage et il conserve toute sa fraîcheur.

\section{IRLANDE}

\section{Situation économique de l'industrie laitière}

Les Services officiels chargés de régulariser le marché des produits laitiers s'inquiètent de l'écart grandissant qui s'établit entre la production du lait en plein développement et la consommation qui reste stable. Il en résulte que les prix accordés aux fermiers devront être probablement réduits.

Pour l'année ayant pris fin le 31 mars 1957, le prix garanti pour l'Irlande du Nord avait été estimé d'après une production de 95 millions de gallons, or la quantité réellement produite a dépassé ce chiffre, de telle sorte que le prix réellement payé aux producteurs a été abaissé de 37,16 d par gallon à 35,12 .

On s'efforce donc de rechercher les moyens d'utiliser au maximum les sous-produits de l'industrie laitière et un facteur encourageant est constitué par le développement de la fabrication de la 
caséine dont la stabilité semble être assurée depuis qu'on a la certitude de disposer d'importantes quantités de lait écrémé.

La production de la caséine en Irlande avait été jusqu'à présent relativement faible, mais on estime qu'elle peut être doublée facilement et que la caséine irlandaise est capable de lutter sur les marchés mondiaux contre la caséine provenant des principaux producteurs.

\section{Exportation de crème}

Les exportations de crème produite dans l'Eire ont pris en 1958 un bon départ, si l'on en juge d'après les statistiques valables pour les quatre premiers mois de l'année. En effet, d'après les chiffres déjà publiés, les livraisons de crème fraîche à destination de la Grande-Bretagne, qui constitue le seul marché disponible, sont passées de 619.000 pounds pour les quatre premiers mois de 1957, à 802.000 pour la période correspondante de 1958 et les exportations de crème de conserve se sont élevées de 168.000 à 212.000 pounds.

Les exportations irlandaises continuent à bénéficier des restrictions apportées par la Grande-Bretagne aux importations de crème fraîche en provenance de pays étrangers à la zone sterling.

En 1957, les exportations de crème fraîche en Grande-Bretagne ont atteint pour l'année entière, 23.100 cwts et celles de crème de conserve 4.800 cwts pour une valeur globale de 450.000 livres sterlings.

\section{NOUVELLE-ZÉLANDE}

\section{Production de beurre et de fromage}

La production totale de beurre pour la période de douze mois, ayant pris fin le 31 juillet 1957, s'est élevée à environ 200.000 tonnes dont 42.000 ont été consommées sur place et 158.000 ont été exportées. La production totale de fromages a atteint environ 92.500 tonnes dont 87.000 ont été vendues à l'étranger. On estime que d'importants changements devront être apportés aux conditions de vente et au mode de fixation des prix pour maintenir la position de l'industrie des produits laitiers en Nouvelle-Zélande.

\section{Industrie de la caséine}

La fabrication de la caséine a été encouragée par une stabilité relative de la production et la plus grande partie de la caséine a été vendue dans des conditions favorables. La caséine de présure trouve un marché plus réduit que celui de la caséine lactique et on envisage de réduire sa production. Pour la saison 1956-1957, il a été produit 9.600 tonnes de caséine lactique, soit $10 \%$ de moins 
que pendant la période annuelle précédente et le prix de vente s'est élevé à environ 180 livres sterling par tonne.

Pour les onze mois prenant fin le 30 juin 1957, la production de poudre de lait écrémé a atteint 49.260 tonnes, supérieure de 10.000 tonnes à celle de la période record de 1953-1954. La production de poudre de petit-lait a plus que doublé au cours des quatre dernières années et a atteint 13.776 tonnes pour la dernière période annuelle au sujet de laquelle on dispose de statistiques.

\section{Modification de la coloration du beurre néo-zélandais}

Le Department of Scientific and Industrial Research a trouvé le moyen de réduire la coloration jaune du beurre néo-zélandais et si cette méthode se révèle utilisable industriellement, elle permettra de trouver de nouveaux marchés pour le produit.

Cette méthode consiste à ajouter à l'alimentation des vaches laitières, deux fois par jour, une certaine quantité de paraffine liquide. Le rendement en lait, la teneur en graisse et en résidu sec du lait, l'indice d'iode et de saponification de la matière grasse du lait ne sont pas modifiés. On enregistre simplement une réduction de la teneur en vitamine $\mathrm{A}$, mais cela ne présente pas de grande importance pour le beurre de Nouvelle-Zélande, très riche en vitamine A, du fait que les vaches sont nourries sur pâturages. Des essais se poursuivent pour vérifier si cette addition de paraffine à la nourriture des animaux n'a pas d'influence sur leur santé.

\section{Développement de la fabrication du fromage sans croûte}

La fabrication du fromage sans croûte en Nouvelle-Zélande se poursuit encore sur une échelle expérimentale, elle a cependant atteint une production de 4.000 tonnes environ en 1956, et 6.000 en 1957.

Les fromages sont emballés en vue de leur exportation dans des boîtes en carton et dans des caisses en bois. Ils peuvent supporter dans ces conditions des transports vers des régions éloignées, mais la principale difficulté qui subsiste est la suppression complète du développement des moisissures sur les surfaces des fromages et lorsque ce problème sera complètement résolu, de nouveaux marchés d'exportation pourront être trouvés.

\section{AUSTRALIE}

\section{Les recherches laitières}

Le récent rapport annuel publié par la Dairy Research Section of C.S.I. R. O. de Melbourne, montre que les chercheurs de cet organisme ont apporté une importante contribution à l'industrie 
de la science et de la technologie laitière. Le but essentiel de cet organisme est d'aider l'industrie laitière australienne à améliorer la qualité de la production, mettre au point de nouveaux produits, réduire les pertes et les frais de fabrication.

Un des principaux problèmes posés avait été la mécanisation complète de la fabrication du fromage. En 1954, une méthode rapide de fabrication de fromage Cheddar par un procédé entièrement mécanisé avait été imaginée. En 1955-1956, les travaux se sont poursuivis et ont permis en 1957 de réaliser une machine qui a été construite et qui fait l'objet actuellement d'études expérimentales. La caillebotte obtenue par cet appareil est légèrement plus fibreuse et retient une plus forte proportion d'humidité que la caillebotte traitée dans les conditions habituelles.

D'autres recherches ont porté sur l'examen de la sensibilité de différents éveilleurs d'acide à la lacténine, à la pénicilline et à la nisine, et sur l'établissement d'un milieu de culture pour éveilleur d'acide susceptible de résister aux bactériophages. Dans le domaine purement scientifique, la microscopie par fluorescence a été appliquée à l'étude de la caséine dans le lait, de la coagulation du lait par la présure, et de la modification de la caillebotte, et du fromage au cours du mûrissage.

Cependant, un des progrès les plus importants réalisés à Melbourne a été la mise au point de deux poudres de lait modifié. L'une de celle-ci dénommée poudre pour meringue peut être employée à la place de l'albumine de l'œuf dans la fabrication de la confiserie, l'autre dénommée poudre spongieuse peut remplacer $90 \%$ des œufs dans la fabrication de la pâtisserie. Le premier de ces produits est déjà fabriqué industriellement et on a pu établir que certains défauts constatés dans la fabrication de ce produit résultaient de fautes de production.

Enfin, un dispositif a été imaginé qui permet, par une installation entièrement automatique, d'enrichir le lait en concentré de vitamine. On utilise dans ce but une solution d'acétate de vitamine A dans l'huile, ce produit est ajouté au lait et permet d'obtenir un lait condensé sucré qui peut se conserver parfaitement pendant neuf mois, à la température ordinaire.

\section{AFRIQUE DU SUD}

\section{Record de production laitière}

En 1957, la production laitière a atteint des chiffres records dans toutes les parties de l'Union Sud-Africaine. Cependant, on craint que la situation ne se maintienne pas par suite des conditions 
atmosphériques moins favorables qu'on a enregistrées depuis cette époque.

Au Natal, la production de crème et de lait a dépassé tous les records enregistrés jusqu'à présent.

\section{Production de beurre}

On a enregistré en 1957, une production de beurre importante, ainsi qu'une production de fromage convenable. Une fabrique de caséine, disposant d'une installation moderne, a été mise en service à Walvis Bay et a commencé sa production en octobre 1957. Cette installation a été réalisée grâce à la collaboration des producteurs de produits laitiers du territoire, sous les auspices du Dairy Industry Control Board et avec la collaboration du Department of Agriculture. Les prix de vente des différentes qualités de caséine ont été fixés par le Board qui se rendra acheteur de la totalité de la production de l'usine.

\section{Stabilisation de l'industrie laitière}

Le Dairy Industry Control Board a créé un Fonds général de stabilisation destiné à venir en aide à l'industrie laitière de l'Afrique du Sud. L'un des buts essentiels de ce Fonds est de combattre les fluctuations des cours qui peuvent résulter des variations saisonnières de la production, de la consommation des usines, et du montant des exportations.

On ne cherchera pas à maintenir des prix rigoureusement stables, ceux-ci devront évoluer comme les prix des principaux produits alimentaires, mais on veut cependant limiter les variations en cours et en particulier faire disparaître celles qui pourraient provenir de manœuvres spéculatives.

\section{INDE}

\section{Le développement de la laiterie}

Lorsqu'on étudie le développement possible de la laiterie en Inde, il ne faut pas perdre de vue que ce pays s'étend sur une longueur de 1.700 miles de l'est à l'ouest, et sur une distance de 2.000 miles du nord au sud. Pour une surface qui est à peu près la moitié de celle des Etats-Unis, la population est le double de la population américaine. L'Inde est avant tout un pays agricole et $83 \%$ de la population se trouvent répartis dans plus de 600.000 villages.

L'agriculture indienne varie énormément suivant les districts, le climat est chaud pendant une grande partie de l'année et son caractère est dominé par l'existence de la mousson qui provoque des pluies abondantes de juin à octobre.

En dehors de quelques établissements gouvernementaux ou 
militaires parfaitement organisés, il n'existe pas d'importantes fermes productrices de lait, et chaque fermier ne dispose que de une ou deux vaches, malgré que le nombre de vaches laitières dépasse deux cents millions dont la production est estimée à environ vingt milliards de litres de lait. La production moyenne par animal est très faible, moins de quatre cents litres de lait contre deux mille cinq cent pour la production moyenne américaine.

Malgré l'importance du nombre d'animaux producteurs, la consommation de lait par habitant est très faible. Pour améliorer l'agriculture, le Gouvernement a institué en 1951, un plan quinquennal dont le but était de rendre le pays indépendant de l'étranger pour son alimentation. Cependant, les sommes prévues pour le développement de l'industrie laitière étaient à cette époque, très réduites, et il a fallu attendre la mise en vigueur du second plan quinquennal pour que cette industrie soit moins sacrifiée.

Le nouveau projet envisage l'organisation du commerce du lait à l'état liquide dans les grands centres urbains, et trente-six villes importantes doivent être équipées d'installations modernes permettant le traitement du lait, de la crème et de la crème glacée, nécessaires aux besoins de leurs populations. Ces installations disposeront également de fabriques de beurre et de ghee destinés à l'utilisation des surplus de lait.

Enfin, on envisage également la création de laiteries rurales qui seront également équipées en vue de la fabrication du beurre, du ghee, de la poudre de lait et de la caséine.

\section{BIBLIOGRAPHIE ANALYTIQUE}

\section{$1^{\circ}$ LES LIVRES}

Jacquot (R.), Le Bars (H.) et Simonnet (H.). - Nutrition animale. Biologie, physiologie et alimentation rationnelle. Volume I : Données générales sur la nutrition et l'alimentation. Nouvelle encyclopédie agricole publiée sous la direction de $J$. Lefevre et de $P$. Tissot. 1 volume broché illustré, $15,5 \times 24,0$ de 472 pages. J.-B. Baillière et fils, éditeurs, 19, rue Hautefeuille. Paris $\left(6^{\mathrm{e}}\right)$. 1958. Prix : 3.900 franes.

Ce livre, destiné plus spécialement aux éleveurs, agriculteurs, zootechniciens, vétérinaires, ingénieurs agronomes et agricoles, qui s'intéressent à la production animale, nous parait venir à son heure, car de données purement scientifiques, il permet de tirer un enseignement pratique important. Il innove car il ne nous semble pas qu'il existe, en langue française, d'ouvrage de synthèse reliant entre eux les enseignements de la physiologie, de la 